\title{
Publisher's Note: Experimental measurements and theoretical model of the cryogenic performance of bialkali photocathode and characterization with Monte Carlo simulation [Phys. Rev. Accel. Beams 19, 103401 (2016)]
}

\author{
Huamu Xie, Ilan Ben-Zvi, Triveni Rao, Tianmu Xin, and Erdong Wang \\ (Received 25 October 2016; published 14 November 2016)
}

DOI: 10.1103/PhysRevAccelBeams.19.119901

This paper was published on 16 October 2016 with an error in the third paragraph in the right-hand column. The paragraph should read "In the second step, the electrons will diffuse with the initial velocity and then experience three possible types of scattering: Electron-electron scattering, electron-hole scattering, and electron-phonon scattering. Here we did not consider the electron-hole recombination in electron transport." The text has been corrected online as of 27 October 2016. 